\title{
MORSE THEORY ON BANACH MANIFOLDS
}

\author{
BY K. UHLENBECK
}

Communicated by Richard Palais, June 23, 1969

S. Smale has conjectured, in an unpublished paper, that the Morse Theory on Hilbert mainfolds due to Palais and Smale [1], [4] can be extended to Banach manifolds. Under a different definition of nondegeneracy of critical points we have been able to make this extension. The result also extends Morse theory on Hilbert manifolds to a wider class of functions. I wish to thank R. Palais for several helpful suggestions.

Let $f$ be a real-valued $C^{1}$ function on a $C^{1}$ Banach manifold $X$. A critical point $x$ of $f$ is said to be weakly nondegenerate if there exists a neighborhood $U$ of $x$ and a hyperbolic linear isomorphism $L_{x}$ : $T_{x}(X) \rightarrow T_{x}(X)$ such that in the coordinate system of $U, d f_{x+v}\left(L_{x} v\right)>0$ for all $x+v$ in $U, v \neq 0$. Then $T_{x}(X)$ splits into the direct sum of two invariant subspaces of $L_{x}, T_{x}(X) \cong T_{x}(X)_{+} \oplus T_{x}(X)_{-}$such that the spectrum of $L_{x}$ on $T_{x}(X)_{+}$lies in the right half plane and the spectrum of $L_{x}$ on $T_{x}(X)_{-}$lies in the left half plane. The index of $f$ at $x$ is defined to be $\operatorname{dim} T_{x}(X)_{-}$, and this term is well defined. A nondegenerate critical point of a function on a Hilbert manifold is weakly nondegenerate.

THEOREM 1. Let $f$ be a $C^{2}$ function on a $C^{2}$ paracompact manifold $X$ without boundary modeled on a separable Banach space $B$. We assume that $B$ has $C^{2}$ partitions of unity and a metric which is $C^{2}$ away from 0 . If, in addition,

(a) $f$ satisfies condition (C) of Palais and Smale with respect to a complete Finsler metric on $X$, and

(b) $q>q^{\prime}$ are not critical values, and all the critical points in $f^{-1}\left(\left(q, q^{\prime}\right)\right)$

are weakly nondegenerate of finite index,

then there exists a homeomorphism $\theta: f^{-1}[q,-\infty) \cong f^{-1}\left[q^{\prime},-\infty\right) \cup h_{i}$ where a handle $h_{i}$ of index $q_{i}$ is added for each one of the finite number of critical points $x_{i} \in f^{-1}\left(\left(q, q^{\prime}\right)\right)$ of index $q_{i}$.

REMARK. In the case of an infinite index, a similar result holds, provided that

$$
d f_{x+v}\left(L_{x} v\right)>\alpha\left(\|v\|_{B}\right) \quad \text { for } \quad 0 \neq v \in T_{x}(M)_{-} \cap U
$$

where $\alpha$ is a continuous function from $R^{+} \rightarrow R^{+}$. 
THEOREM 2. Let $\eta$ be a vector bundle over a finite dimensional manifold $N$. Let the integral $J: L_{\boldsymbol{k}}^{p}(\eta)_{0} \rightarrow R$ be given by

$$
J(s)=\int_{N}\left(1+|A(s)|^{2}\right)^{p / 2}+B(s) d u \quad(p \geqq 2)
$$

where $A$ is a nonlinear (over-determined) elliptic system of order and weight $k, p k>\operatorname{dim} N$, and $B$ is of order $k-1$ and weight pk. ${ }^{1}$ Then $J$ is $C^{[p]}\left(C^{\infty}\right.$ for $p$ even) on the Sobolev space $L_{\mathbf{k}}^{p}(\eta)_{0}$, and if the critical point $v$ has the properties:

(a) $v \in C^{k+\alpha}(\eta)$ for any $\alpha>0$,

(b) the bilinear form $d^{2} J_{v}($,$) extends to a nondegenerate form on$ $H_{k}(\eta)_{0}$,

then $v$ is a weakly nondegenerate critical point of $J$ with finite index.

\section{REFERENCES}

1. R. S. Palais, Morse theory on Hilbert manifolds, Topology 2 (1963), 299-340. MR 28 \#1633.

2. - Ljusternik-Schnirelmann theory on Banach manifolds, Topology, 5 (1966), 115-132.

3. - Foundations of non-linear analysis, Benjamin, New York, 1968.

4. S. Smale, Morse theory and a non-linear generalization of the Dirichlet problem, Ann. of Math. (2) 80 (1964), 382-396. MR 29 \#2820.

5. - Morse theory on Finsler manifolds (unpublished article).

Massachusetts Institute of Technology, Cambridge, Massachusetts 02139

${ }^{1}$ See Chapter 16 of [3]. 